\title{
Inhomogeneous plane waves in elastic materials with voids *
}

\author{
Stan Chiriţă \\ Faculty of Mathematics, Al. I. Cuza University of Iaşi, Blvd. Carol I, no. 11, \\ 700506 - Iaşi, Romania \\ Ionel-Dumitrel Ghiba \\ "Octav Mayer" Mathematics Institute, Romanian Academy of Science, Iaşi \\ Branch, Blvd. Carol I, no. 8, 700505 - Iaşi, Romania \&5 \\ Faculty of Mathematics, Al. I. Cuza University of Iaşi
}

\begin{abstract}
In this paper we present inhomogeneous plane wave solutions within the context of linear theory of poroelastic materials. We consider the class of strongly elliptic homogeneous poroelastic materials with a center of symmetry. To construct the solutions in concern, we use the "directional-ellipse"method, which reduces the propagation problem to a secular equation for wave speeds and gives further on the algebraic system which furnishes the slowness and amplitude bivectors. Explicit expressions for all possible inhomogeneous plane waves are presented in the case of isotropic poroelastic materials. These solutions are further used to study the Rayleigh waves in an isotropic poroelastic half-space. The explicit equation for the Rayleigh surface wave speed is established.
\end{abstract}

Key words: Inhomogeneous plane waves, Elastic materials with voids, Slowness bivector, Directional-ellipse method, Rayleigh waves

2000 Mathematics Subject Classification: 74E20, 74J05, 74J15.

\footnotetext{
ऋ The authors are very grateful to the reviewers for useful observations. They also acknowledge support from the Romanian Ministry of Education and Research, CNCSIS Grant code ID-401, Contract no. 15/28.09.2007.

Email addresses: schirita@uaic.ro (Stan Chiriţă), ghiba_dumitrel@yahoo.com (Ionel-Dumitrel Ghiba).
} 


\section{Introduction}

The propagation of elliptically polarised inhomogeneous time-harmonic plane waves has applications in many areas including Rayleigh, Love and Stoneley waves in classical linear elasticity theory. The inhomogeneous plane waves are known as evanescent waves and they represent those waves for which the planes of constant phase are not the same as the planes of constant amplitude. These types of waves are described in terms of two complex vectors or bivectors: the slowness bivector and the amplitude bivector. Hayes [1] has developed the directional-ellipse method for a systematic study of all inhomogeneous plane waves that may propagate in the classical linear elasticity. By means of this method, in classical elasticity, the propagation problem leads to an eigenvalue problem for a complex $3 \times 3$ matrix, namely the complex symmetric acoustical tensor [2].

On the other hand, wave propagation in an elastic material containing voids is an area with immense potential for practical applications. Many theories which describe the behaviour of materials with voids are developed and they proved to be useful in practice. In seismic zones buildings are constructed with much lighter porous materials and typically have thinner walls. As a consequence, there is a great need to study the acoustic properties of porous materials including the nature of the solid elastic matrix and the gas filling the pores, and the influence of temperature on these quantities. But seismology represents only one of the many fields where the theory of materials with voids is applied. Medicine, various branches of biology and oil exploration industry are other important fields of application.

In this paper we consider the theory of materials with voids introduced by Cowin \& Nunziato [3]. The theory of elastic materials with voids is the simplest extension of the classical theory of elasticity. The basic idea of this theory is to suppose that there is a distribution of voids throughout the elastic body. Consequently, the bulk density is written as the product of two fields: the matrix material density field and the volume fraction field. This representation introduces an additional degree of kinematic freedom and it was employed previously by Goodman \& Cowin [4] to develop a continuum theory of granular materials. The first investigations in the theory of thermoelastic materials with voids are due to Nunziato \& Cowin [5] and Ieşan [6]. The Nunziato-Cowin theory has enjoyed much success in predicting various effects especially within the remit of linear theory, see e.g. Ciarletta \& Ieşan [7], Ieşan [8] and the recent work of Quintanilla [9], Casas \& Quintanilla [10], Magaña \& Quintanilla [11], [12] and Chiriţă \& Ghiba [13] and the references therein. The intended applications of the theory concern the manufacture of building materials, e.g. plasterboard, brick, concrete.

The purpose of this paper is to study the inhomogeneous plane wave solutions for the equations of motion within the framework of the linear theory of poroelastic materials. We consider a strongly elliptic homogeneous poroelastic material with a center of symmetry. Then we use the directional-ellipse method developed by Hayes [1] in order to obtain the generalized form of the secular equation and the algebraic system which furnishes 
information upon the slowness bivectors and the corresponding amplitude bivectors. Thus, the propagation problem is reduced to an algebraic equation for wave speed. Explicit expressions for all possible inhomogeneous plane waves are further obtained for strongly elliptic isotropic elastic materials with voids. Finally, these inhomogeneous plane wave solutions are used to study the Rayleigh surface waves in an isotropic poroelastic halfspace. An explicit equation is also established for the Rayleigh wave speed.

We have to outline that the problem of homogeneous and acceleration waves propagation in materials with voids was studied in various works (see, e.g. Nunziato \& Walsh [14], [15]; Nunziato et al. [16]; Cowin \& Nunziato [3]; Ciarletta \& Straughan [17], [18]; Ciarletta et al. [19]) and a comprehensive review of the results can be found in the book by Straughan [20]. The use of acceleration waves and related analysis have proved extremely useful in recent investigations of wave motion in various dispersive and random media, in a variety of thermodynamic states, see e.g. Ostoja-Starzewski \& Trebicki [21], Puri \& Jordan [22], Quintanilla \& Straughan [23], Christov \& Jordan [24], Jordan \& Christov [25]; Jordan \& Puri [26], Christov et al. [27]. In [13] we have studied the non dispersive behaviour of homogeneous progressive waves. In the present paper we study the dispersive characteristic behaviour of inhomogeneous plane waves. We show how the theory of complex vectors, or bivectors as Hamilton and Gibbs called them, may be used in the study of inhomogeneous plane waves propagation in poroelastic materials. The use of the directional-ellipse method leads to a simple direct formulation of an algebraic equation for the wave speed of these waves.

The present mathematical results can be used in laboratories for studying the specific properties of materials with voids.

\section{Basic equations. Preliminary results}

Throughout this section $B$ represents a bounded regular region of the three-dimensional Euclidean space. We denote by $\bar{B}$ the closure of $B$ and by $\partial B$ the boundary of $B$. We assume that the body occupying $B$ is a linearly elastic centro-symmetric solid with voids. The body is referred to a fixed system of rectangular Cartesian axes $O x_{i}(i=1,2,3)$. Throughout this paper Latin indices have the range 1, 2, 3 and the usual summation convention is employed. Typical conventions for differential operations are implied such as a superposed dot or comma followed by a subscript to denote the partial derivative with respect to the time or the corresponding cartesian coordinate variable.

In this paper we use the following notations:

i) $\mathbf{u}$ is the displacement field over $B$;

ii) $\varphi$ is the volume fraction field over $B$;

iii) $t_{r s}$ are the components of the stress tensor;

iv) $h_{r}$ are the components of the equilibrated stress vector; 
v) $g$ is the intrinsic equilibrated body force;

vi) $\varrho$ is the density mass;

vii) $\varkappa$ is the equilibrated inertia.

The components $e_{r s}$ of the linear strain tensor are given by

$$
e_{r s}=\frac{1}{2}\left(u_{r, s}+u_{s, r}\right)
$$

and the constitutive equations for a homogeneous and anisotropic centro-symmetric elastic material with voids are [3]

$$
\begin{aligned}
t_{r s} & =C_{r s m n} e_{m n}+B_{r s} \varphi, \\
h_{r} & =A_{r s} \varphi_{, s}, \\
g & =-B_{r s} e_{r s}-\xi \varphi,
\end{aligned}
$$

where $A_{r s}, B_{r s}, C_{r s m n}$ and $\xi$ are constant constitutive coefficients satisfying the following symmetries

$$
A_{r s}=A_{s r}, \quad B_{r s}=B_{s r}, \quad C_{m n r s}=C_{r s m n}=C_{n m r s} .
$$

The specific internal energy $W$ is given by

$$
W(\mathbf{e}, \varphi, \operatorname{grad} \varphi)=\frac{1}{2} C_{m n r s} e_{m n} e_{r s}+\frac{1}{2} \xi \varphi^{2}+\frac{1}{2} A_{r s} \varphi_{, r} \varphi_{, s}+B_{r s} e_{r s} \varphi .
$$

The specific internal energy $W_{0}$ for an isotropic and homogeneous elastic material with voids is defined by

$$
W_{0}(\mathbf{e}, \varphi, \operatorname{grad} \varphi)=\mu e_{r s} e_{r s}+\frac{1}{2} \lambda e_{r r} e_{s s}+\frac{1}{2} \xi \varphi^{2}+\frac{1}{2} \alpha \varphi_{, r} \varphi_{, r}+\beta \varphi e_{r r},
$$

where $\lambda, \mu, \alpha, \beta$ and $\xi$ are constant constitutive coefficients.

We will say that the poroelastic material is strongly elliptic if

$$
W(\mathbf{A}, \eta, \boldsymbol{\zeta})>0, \quad \text { for all }(\mathbf{A}, \varphi, \boldsymbol{\zeta}) \neq \mathbf{0}
$$

with $\mathbf{A}=\mathbf{m} \otimes \mathbf{n}, \mathbf{m}, \mathbf{n}$ and $\boldsymbol{\zeta}$ arbitrary vectors and $\eta$ an arbitrary scalar.

The strong ellipticity condition has been studied by Chiriţă \& Ghiba [13]. They prove that a centro-symmetric elastic material with voids is strongly elliptic if and only if $A_{r s}$ is a positive definite tensor, $\xi>0$ and $\mathcal{C}_{m n r s}=\xi C_{m n r s}-B_{m n} B_{r s}$ is a strongly elliptic tensor, that is,

$$
\mathcal{C}_{m n r s} \zeta_{m} \zeta_{r} \eta_{n} \eta_{s}>0 \text { for all non-zero vectors } \boldsymbol{\zeta}, \boldsymbol{\eta}
$$

In the absence of the body force and the extrinsic equilibrated body force, the equations of motion of the elastic material with voids are given by [3]

$$
\begin{aligned}
t_{r s, r} & =\varrho \ddot{u}_{s}, \\
h_{r, r}+g & =\varrho \varkappa \ddot{\varphi} .
\end{aligned}
$$


If we substitute (1) and (2) into (4) we obtain the basic equations in terms of the displacement and volume fraction fields in the form

$$
\begin{aligned}
C_{r s m n} u_{m, n r}+B_{r s} \varphi_{, r} & =\varrho \ddot{u}_{s}, \\
A_{m n} \varphi_{, n m}-B_{r s} u_{r, s}-\xi \varphi & =\varrho \varkappa \ddot{\varphi} .
\end{aligned}
$$

For isotropic materials with voids the equations of motions (5) become

$$
\begin{aligned}
\mu \Delta u_{r}+(\lambda+\mu) u_{s, s r}+\beta \varphi_{, r} & =\varrho \ddot{u}_{r}, \\
\alpha \Delta \varphi-\xi \varphi-\beta u_{r, r} & =\varrho \varkappa \ddot{\varphi},
\end{aligned}
$$

where $\Delta$ is the Laplace operator.

The isotropic elastic material with voids is strongly elliptic if and only if the elastic coefficients satisfy the following inequalities (see [13])

$$
\begin{aligned}
\mu & >0, \\
\xi & >0, \\
\alpha & >0, \\
\xi(\lambda+2 \mu) & >\beta^{2} .
\end{aligned}
$$

Throughout this paper we will assume that the elastic material with voids is strongly elliptic and, therefore, the conditions (3) and (7) hold true.

\section{Inhomogeneous plane waves}

In this section we deduce the equations for the slowness and for the amplitude of inhomogeneous plane waves. In what follows, the superscripts + and - denote the real and imaginary parts of a complex quantity. Thus, we consider solutions of (5) in the form

$$
\begin{aligned}
& \mathbf{u}=\mathbf{A} \exp [\mathrm{i} \omega(\mathbf{S} \cdot \mathbf{x}-\mathrm{t})] \\
& \varphi=\phi \exp [\mathrm{i} \omega(\mathbf{S} \cdot \mathbf{x}-\mathrm{t})]
\end{aligned}
$$

where $\omega$ is a real representing the angular frequency, $\mathbf{S}=\mathbf{S}^{+}+\mathrm{i} \mathbf{S}^{-}$is the slowness bivector (see [1] or [2]), $\mathbf{A}=\mathbf{A}^{+}+\mathbf{i A}^{-}$is the amplitude bivector and $\mathrm{i}=\sqrt{-1}$ is the complex unit. The relation (8) represents an infinite train of elliptically polarised plane waves. The waves travel in the direction of $\mathbf{S}^{+}$with slowness $\left|\mathbf{S}^{+}\right|$and they are attenuated in the direction of $\mathbf{S}^{-}$. For any fixed $\mathbf{x}$ the displacement vector $\mathbf{u}^{+}$lies on an ellipse which is similar and similarly situated to the ellipse of $\mathbf{A}$, namely the ellipse whose conjugate semi-diameters are $\mathbf{A}^{+} \exp \left(-\omega \mathbf{S}^{-} \cdot \mathbf{x}\right)$ and $\mathbf{A}^{-} \exp \left(-\omega \mathbf{S}^{-} \cdot \mathbf{x}\right)$. 
A solution of the form (8) is called an inhomogeneous plane wave when $\mathbf{S}^{-}$is not parallel to $\mathbf{S}^{+}$. When $\mathbf{S}^{-}$is parallel to $\mathbf{S}^{+}$we have attenuated homogeneous plane waves.

The planes $\mathbf{S}^{+} \cdot \mathbf{x}=$ constant are called planes of constant phase and the planes $\mathbf{S}^{-} \cdot \mathbf{x}=$ constant are called the planes of constant amplitude. We observe that in the case of an inhomogeneous plane wave, the planes of constant amplitude are different from the planes of constant phase. The phase speed is $v=\left|\mathbf{S}^{+}\right|^{-1}$ and $\left|\mathbf{S}^{-}\right|$is the attenuation coefficient. If $\mathbf{A} \cdot \mathbf{S}=0$ then the wave is called transverse and if $\mathbf{A} \times \mathbf{S}=\mathbf{0}$ then the wave is called longitudinal.

In the directional-ellipse method developed by Hayes [1] and Boulanger and Hayes [2], the slowness bivector $\mathbf{S}$ is written in the form

$$
\mathbf{S}=N \mathbf{C}
$$

where $N$ is a complex number and $\mathbf{C}$ is so-called directional bivector and it has the form

$$
\mathbf{C}=q \hat{\mathbf{m}}+\mathrm{i} \hat{\mathbf{n}}, \quad \hat{\mathbf{m}} \cdot \hat{\mathbf{n}}=0, \quad|\hat{\mathbf{m}}|=|\hat{\mathbf{n}}|=1, \quad q \geq 1 .
$$

The direction of the unit vector $\hat{\mathbf{n}}$, the magnitude $q$ and direction of $\hat{\mathbf{m}}$, which is orthogonal to $\hat{\mathbf{n}}$, are specified.

By the directional-ellipse method, prescribing the directional bivector $\mathbf{C}=q \hat{\mathbf{m}}+\mathrm{i} \hat{\mathbf{n}}$, we can determine the corresponding value for $N$ and then, consequently, we can determine the slowness $\mathbf{S}$ and the amplitude bivector $\mathbf{A}$.

If we substitute the plane inhomogeneous wave described by (8) into basic equations (5) and then we take into consideration the assumptions described into (9) and (10), we can see that such waves are possible in an elastic material with voids if and only if

$$
\begin{aligned}
\left(N^{2} C_{r s m n} C_{n} C_{r}-\varrho \delta_{m s}\right) \omega^{2} A_{m}-\mathrm{i} N \omega B_{r s} C_{r} \phi & =0, \\
\mathrm{i} N \omega B_{r s} C_{s} A_{r}+\left[\left(N^{2} A_{m n} C_{m} C_{n}-\varrho \varkappa\right) \omega^{2}+\xi\right] \phi & =0 .
\end{aligned}
$$

Such linear algebraic system admits a non-zero solution $\left(A_{1}, A_{2}, A_{3}, \phi\right)$ if and only if $N$ satisfies the following algebraic equation

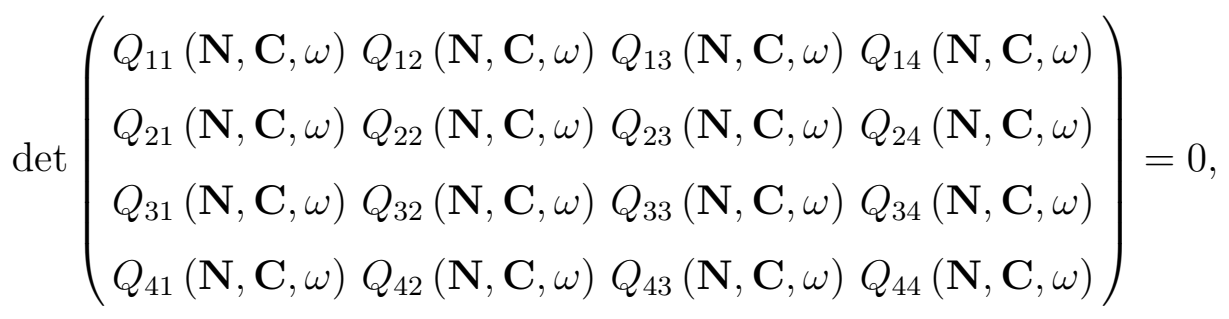


where

$$
\begin{aligned}
Q_{s m}(\mathbf{N}, \mathbf{C}, \omega) & =\left(N^{2} C_{r s m n} C_{n} C_{r}-\varrho \delta_{s m}\right) \omega^{2}, \\
Q_{s 4}(\mathbf{N}, \mathbf{C}, \omega) & =-Q_{4 s}(\mathbf{N}, \mathbf{C}, \omega)=-\mathrm{i} N \omega B_{r s} C_{r}, \\
Q_{44}(\mathbf{N}, \mathbf{C}, \omega) & =\left(N^{2} A_{r s} C_{r} C_{s}-\varrho \varkappa\right) \omega^{2}+\xi .
\end{aligned}
$$

Thus, for any prescribed directional bivector $\mathbf{C}=q \hat{\mathbf{m}}+\mathbf{i} \hat{\mathbf{n}}$, the values of the complex number $N$ are obtained by solving the generalized form (12) of the secular equation, while the corresponding non-zero solutions $\left(A_{1}, A_{2}, A_{3}, \phi\right)$ are obtained by solving the corresponding system (11). We have to note that we retrieve the case of homogeneous plane waves by taking $\mathbf{C}$ to be a unit vector $\mathbf{n}$ along the propagation direction. Then, $N^{-2}$ becomes the squared wave speed of homogeneous plane waves propagating along $\mathbf{n}$ [13].

\section{Isotropic poroelastic materials}

In this section we find inhomogeneous plane wave solutions for the equations of motion (6).

To this end we note that (13) becomes

$$
\begin{aligned}
Q_{s m}(\mathbf{N}, \mathbf{C}, \omega) & =\left[N^{2}(\lambda+\mu) C_{s} C_{m}+\left(\mu N^{2} C_{r} C_{r}-\varrho\right) \delta_{s m}\right] \omega^{2}, \\
Q_{s 4}(\mathbf{N}, \mathbf{C}, \omega) & =-Q_{4 s}(\mathbf{N}, \mathbf{C}, \omega)=-\mathrm{i} N \omega \beta C_{s}, \\
Q_{44}(\mathbf{N}, \mathbf{C}, \omega) & =\left(N^{2} \alpha C_{r} C_{r}-\varrho \varkappa\right) \omega^{2}+\xi
\end{aligned}
$$

and the equation (12) gives the following generalized form of the secular equation

$$
\left(\mu X^{2}-\varrho \omega^{2}\right)^{2}\left\{\left(\alpha X^{2}+\xi-\varrho \varkappa \omega^{2}\right)\left[(\lambda+2 \mu) X^{2}-\varrho \omega^{2}\right]-\beta^{2} X^{2}\right\}=0
$$

where

$$
X^{2}=\omega^{2}(\mathbf{C} \cdot \mathbf{C}) N^{2}
$$

This equation has the double root given by

$$
\mu X^{2}-\varrho \omega^{2}=0
$$

and the corresponding amplitude and slowness bivectors are orthogonal, that is

$$
\mathbf{A} \cdot \mathbf{S}=0 .
$$

So this corresponds to the transverse wave.

The other two roots are given by

$$
\alpha(\lambda+2 \mu) X^{4}+\left[\xi(\lambda+2 \mu)-\beta^{2}-(\alpha+\varkappa(\lambda+2 \mu)) \varrho \omega^{2}\right] X^{2}-\varrho \omega^{2}\left(\xi-\varrho \varkappa \omega^{2}\right)=0,
$$


and the corresponding amplitude and slowness bivectors are parallel, that is

$$
\mathrm{A} \times \mathrm{S}=\mathbf{0}
$$

In the linear theory of elastic materials with voids there is the coefficient $\beta$ which couples the equations describing the elastic behaviour of the material with the porosity equation. When $\beta=0$ we have the uncoupled case and the study of equations which arise is similar with the studies presented in [1] and [2, pages 116-124]. In what follows we will consider the propagation of inhomogeneous waves in the coupled case $(\beta \neq 0)$. In this case we will discuss how the effect of porosity affects the elastic inhomogeneous waves and vice versa.

\subsection{Transverse waves}

Corresponding to the double root

$$
X^{2}=\frac{\varrho \omega^{2}}{\mu}
$$

we have

$$
\phi=0
$$

and in consequence we can say that the corresponding transverse wave is not affected by the presence of voids.

We remark that, for propagating transverse waves, the bivector $\mathbf{C}$ may not be isotropic (this means that $\mathbf{C} \cdot \mathbf{C} \neq 0$ ). From (7), (16) and (21) we deduce that, in the case of transverse waves, the plane of constant amplitude is orthogonal to the plane of constant phase $\left(\mathbf{S}^{+} \cdot \mathbf{S}^{-}=0\right)$.

As in classical elasticity [2], we choose

$$
\mathbf{A}=\delta \mathbf{C}_{\perp}+\gamma \hat{\mathbf{m}} \times \hat{\mathbf{n}},
$$

where

$$
\mathbf{C}_{\perp}=q^{-1} \hat{\mathbf{m}}+\mathrm{i} \hat{\mathbf{n}}
$$

is the reciprocal of the bivector $\mathbf{C}$ and $\delta$ and $\gamma$ are arbitrary scalars. Hence, for a given non-isotropic directional bivector $\mathbf{C}$, the displacement is

$$
\mathbf{u}=\left[\delta\left(q^{-1} \hat{\mathbf{m}}+\mathrm{i} \hat{\mathbf{n}}\right)+\gamma \hat{\mathbf{m}} \times \hat{\mathbf{n}}\right] \exp \left[\mathrm{i} \omega\left( \pm \sqrt{\frac{\varrho}{\mu\left(q^{2}-1\right)}} q \hat{\mathbf{m}} \cdot \mathbf{x}-t\right)\right] \exp \left(\mp \omega \sqrt{\frac{\varrho}{\mu\left(q^{2}-1\right)}} \hat{\mathbf{n}} \cdot \mathbf{x}\right)
$$


If we take $q \rightarrow \infty$ in the above relation, then the attenuation effect is vanishing and

$$
\mathbf{S}= \pm \sqrt{\frac{\varrho}{\mu}} \hat{\mathbf{m}}
$$

We obtain in this way the ordinary unattenuated transverse wave.

All remarks regarding the transverse inhomogeneous waves from classical elasticity [1] are also true for the propagation of transverse inhomogeneous waves in poroelastic materials. Let us remind just the fact that the wave is circular polarized when the scalars $\delta$ and $\gamma$ are such that

$$
\gamma \delta^{-1}= \pm \sqrt{q^{2}-1} q^{-1}
$$

Thus, in the case of circularly polarized waves, we have the amplitude [2]

$$
\mathbf{A}=\delta\left(q^{-1} \hat{\mathbf{m}}+\mathrm{i} \hat{\mathbf{n}} \pm \sqrt{q^{2}-1} q^{-1} \hat{\mathbf{m}} \times \hat{\mathbf{n}}\right)
$$

\subsection{Longitudinal waves}

In this subsection, we study the inhomogeneous waves corresponding to the other two roots of the equation (15). Thus, we consider the solutions of the equation (19), that is

$$
X_{1,2}^{2}=T_{1,2}\left(\omega^{2}\right),
$$

where

$$
\begin{aligned}
T_{1,2}\left(\omega^{2}\right) & =\frac{1}{2 \alpha(\lambda+2 \mu)}\left\{\beta^{2}-\left(\xi-\varrho \varkappa \omega^{2}\right)(\lambda+2 \mu)+\alpha \omega^{2} \varrho\right. \\
& \left.\mp \sqrt{4 \alpha \omega^{2} \beta^{2} \varrho+\left[-\beta^{2}+\alpha \omega^{2} \varrho+\left(\xi-\varrho \varkappa \omega^{2}\right)(\lambda+2 \mu)\right]^{2}}\right\} .
\end{aligned}
$$

We define the quantity

$$
\omega_{2}^{2}=\frac{\xi}{\varrho \varkappa} .
$$

For $\omega^{2}<\omega_{2}^{2}$, we have

$$
T_{1}\left(\omega^{2}\right)<0, \quad T_{2}\left(\omega^{2}\right)>0,
$$

while for $\omega^{2}>\omega_{2}^{2}$ we have

$$
T_{1}\left(\omega^{2}\right)>0, \quad T_{2}\left(\omega^{2}\right)>0 .
$$


Moreover, we can view that

$$
T_{1}\left(\omega_{2}^{2}\right)=0, T_{2}\left(\omega_{2}^{2}\right)=\frac{\xi \alpha+\beta^{2} \varkappa}{\alpha \varkappa(\lambda+2 \mu)}>0 .
$$

The wave solutions corresponding to (8) and (29) represent longitudinal waves.

If $\omega^{2} \neq \omega_{2}^{2}$ then, for propagating longitudinal waves the bivector $\mathbf{C}$ may not be isotropic.

If $\omega^{2}=\omega_{2}^{2}$ and the bivector $\mathbf{C}$ is not isotropic, then we have only an admissible value of $N^{2}$ corresponding to $T_{2}\left(\omega_{2}^{2}\right)$.

If $\omega^{2}=\omega_{2}^{2}$ and the bivector $\mathbf{C}$ is isotropic then, in view of (16), the admissible values of $N^{2}$ correspond to $T_{1}\left(\omega_{2}^{2}\right)=0$. In this case $N \neq 0$ can be arbitrary and, up to a multiplying scalar factor, the corresponding amplitude is $\mathbf{A}=\frac{\mathrm{i} \beta N}{\varrho \omega} \mathbf{C}$ and $\phi=-1$. We can remark that the amplitude $\mathbf{A}$ depends only on $\beta$ and $\varrho$, while the porosity becomes independent of the material characteristics.

In what follows we consider that $\mathbf{C}$ is not isotropic.

In conclusion, we have

$$
\begin{gathered}
N_{1}=\left\{\begin{array}{c}
\frac{1}{\omega \sqrt{q^{2}-1}} \sqrt{T_{1}\left(\omega^{2}\right)}, \text { if } \omega^{2}>\omega_{2}^{2} \\
\frac{i}{\omega \sqrt{q^{2}-1}} \sqrt{-T_{1}\left(\omega^{2}\right)}, \text { if } \omega^{2}<\omega_{2}^{2}
\end{array}\right. \\
N_{2}=\frac{1}{\omega \sqrt{q^{2}-1}} \sqrt{T_{2}\left(\omega^{2}\right)} .
\end{gathered}
$$

For these values of $N$, we find that, up to a multiplying scalar factor, the amplitude is given by

$$
\mathbf{A}^{(1,2)}=\frac{\mathrm{i} \beta N_{1,2}}{\varrho \omega} \mathbf{C}
$$

and

$$
\phi^{(1,2)}=N_{1,2}^{2} \frac{\lambda+2 \mu}{\varrho}\left(q^{2}-1\right)-1
$$

From (16) and (29) we remark that, for every frequency $\omega$ the longitudinal waves are so that the plane of constant amplitude is orthogonal to the plane of constant phase.

To obtain the case of unattenuated wave we take $q \rightarrow \infty$ in relations (36), (37) and 
in the expression of $\mathbf{S}$. Thus we have

$$
\begin{gathered}
\mathbf{A}^{(1)}=\left\{\begin{array}{l}
\frac{\mathrm{i} \beta}{\varrho \omega^{2}} \sqrt{T_{1}\left(\omega^{2}\right)} \hat{\mathbf{m}}, \text { if } \omega^{2}>\omega_{2}^{2} \\
\frac{\mathrm{i} \beta}{\varrho \omega^{2}} \sqrt{-T_{1}\left(\omega^{2}\right)} \hat{\mathbf{m}}, \text { if } \omega^{2}<\omega_{2}^{2}
\end{array},\right. \\
\phi^{(1)}=\frac{\lambda+2 \mu}{\varrho \omega^{2}} T_{1}\left(\omega^{2}\right)-1, \\
\mathbf{S}^{(1)}=\left\{\begin{array}{l}
\frac{1}{\omega} \sqrt{T_{1}\left(\omega^{2}\right)} \hat{\mathbf{m}}, \text { if } \omega^{2}>\omega_{2}^{2} \\
\frac{\mathrm{i}}{\omega} \sqrt{-T_{1}\left(\omega^{2}\right)} \hat{\mathbf{m}}, \text { if } \omega^{2}<\omega_{2}^{2}
\end{array}\right.
\end{gathered}
$$

and

$$
\begin{aligned}
& \mathbf{A}^{(2)}=\frac{\mathrm{i} \beta}{\varrho \omega^{2}} \sqrt{T_{2}\left(\omega^{2}\right)} \hat{\mathbf{m}}, \phi^{(2)}=\frac{\lambda+2 \mu}{\varrho \omega^{2}} T_{2}\left(\omega^{2}\right)-1, \\
& \mathbf{S}^{(2)}=\frac{1}{\omega} \sqrt{T_{2}\left(\omega^{2}\right)} \hat{\mathbf{m}} .
\end{aligned}
$$

\section{Rayleigh waves}

In this section we consider that the material with voids occupying the half-space $x_{3} \geq 0$ is homogeneous and isotropic. We assume that the body force and the equilibrated force are absent and the boundary $x_{3}=0$ is traction free and equilibrated traction free, that is we assume

$$
t_{i 3}=0, \quad h_{3}=0 \quad \text { for } x_{3}=0 .
$$

Moreover, we require that the solutions be attenuated in the direction $x_{3}$, so that they are decaying with distance from the plane surface $x_{3}=0$, that is we require that

$$
\lim _{x_{3} \rightarrow \infty}\left(u_{1}, u_{2}, u_{3}, \varphi\right)=0 .
$$

Without loss in generality we will study the waves propagating along the $x_{1}$-axis.

We will treat the above problem by means of the inhomogeneous plane waves. Thus, we seek solutions of the above free half-space problem of the form (see, for example, [28])

$$
\begin{aligned}
\mathbf{u} & =\left\{\mathbf{A} \exp \left(-\sigma x_{3}\right) \exp \left[\mathrm{i} \kappa\left(x_{1}-c t\right)\right]\right\}^{+}, \\
\varphi & =\left\{\phi \exp \left(-\sigma x_{3}\right) \exp \left[\mathrm{i} \kappa\left(x_{1}-c t\right)\right]\right\}^{+}, \quad \sigma>0, \kappa>0 .
\end{aligned}
$$

According with the equation (8), by (43) we have

$$
\omega=\kappa c
$$


and the slowness bivector is given by

$$
\omega \mathbf{S}=\kappa \mathbf{i}_{1}+\mathrm{i} \sigma \mathbf{i}_{3}
$$

Then, in view of the relations (9), (10), (16) and (44), we have

$$
X^{2}=\kappa^{2}-\sigma^{2} .
$$

Corresponding to the double root given in $(21)$, by (45) we have for the $S$-wave solution

$$
\sigma_{s}^{2}=\kappa^{2}\left(1-\frac{c^{2}}{v_{s}^{2}}\right)
$$

where

$$
v_{s}=\sqrt{\frac{\mu}{\varrho}} .
$$

On the other hand, by means of relations (18) and (44), we get

$$
A_{1}^{(s)}=-\mathrm{i} \sigma_{s}, \quad A_{2}^{(s)}=0, \quad A_{3}^{(s)}=\kappa .
$$

Thus, using (22) we deduce that, up to a multiplying scalar, the $S$-wave solution is given by

$$
\begin{aligned}
& \mathbf{u}^{(s)}=\left\{\mathbf{A}^{(s)} \exp \left(-\sigma_{s} x_{3}\right) \exp \left[\mathrm{i} \kappa\left(x_{1}-c t\right)\right]\right\}^{+}, \\
& \phi^{(s)}=0
\end{aligned}
$$

where

$$
\mathbf{A}^{(s)}=-\mathrm{i} \sigma_{s} \mathbf{i}_{1}+\kappa \mathbf{i}_{3} .
$$

In the case of $P$-wave solutions, in view of $(29)$ we obtain

$$
\sigma_{p_{1,2}}^{2}=\kappa^{2}\left(1-\frac{T_{1,2}}{\kappa^{2}}\right),
$$

and in consequence, using (36) and (37), we have that, up to a multiplying scalar, the $P$-wave solutions are given by

$$
\begin{aligned}
& \mathbf{u}^{\left(p_{1,2}\right)}=\left\{\mathbf{A}^{\left(p_{1,2}\right)} \exp \left(-\sigma_{p_{1,2}} x_{3}\right) \exp \left[\mathrm{i} \kappa\left(x_{1}-c t\right)\right]\right\}^{+}, \\
& \phi^{\left(p_{1,2}\right)}=\left\{\left[\varrho c^{2} \kappa^{2}-(\lambda+2 \mu)\left(\kappa^{2}-\sigma_{p_{1,2}}^{2}\right)\right] \exp \left[\mathrm{i} \kappa\left(x_{1}-c t\right)\right]\right\}^{+},
\end{aligned}
$$

where

$$
\mathbf{A}^{\left(p_{1,2}\right)}=-\mathrm{i} \kappa \beta \mathbf{i}_{1}+\sigma_{p_{1,2}} \beta \mathbf{i}_{3} .
$$


In order to solve the problem of the free half-space, we seek the solution as a linear combination of the above waves, that is we assume that

$$
\begin{aligned}
& u_{1}=\left\{\left(-\mathrm{i} \sigma_{s} B_{1} \mathrm{e}^{-\sigma_{s} x_{3}}-\mathrm{i} \kappa \beta B_{2} \mathrm{e}^{-\sigma_{p_{1}} x_{3}}-\mathrm{i} \kappa \beta B_{3} \mathrm{e}^{-\sigma_{p_{2}} x_{3}}\right) \mathrm{e}^{\mathrm{i} \kappa\left(x_{1}-c t\right)}\right\}^{+}, \\
& u_{2}=0 \\
& u_{3}=\left\{\left(\kappa B_{1} \mathrm{e}^{-\sigma_{s} x_{3}}+\sigma_{p_{1}} \beta B_{2} \mathrm{e}^{-\sigma_{p_{1}} x_{3}}+\sigma_{p_{2}} \beta B_{3} \mathrm{e}^{-\sigma_{p_{2}} x_{3}}\right) \mathrm{e}^{\mathrm{i} \kappa\left(x_{1}-c t\right)}\right\}^{+}, \\
& \phi=\left\{\left[B_{2}\left[\varrho c^{2} \kappa^{2}-(\lambda+2 \mu)\left(\kappa^{2}-\sigma_{p_{1}}^{2}\right)\right]+B_{3}\left[\varrho c^{2} \kappa^{2}-(\lambda+2 \mu)\left(\kappa^{2}-\sigma_{p_{2}}^{2}\right)\right]\right] \mathrm{e}^{\mathrm{i} \kappa\left(x_{1}-c t\right)}\right\}^{+}
\end{aligned}
$$

where $\kappa$ is a prescribed real constant, $c$ is the wave speed and $B_{i}, \quad i=1,2,3$ are unknown parameters. In view of the above discussion such solution verifies identically the equations of motion. We have to determine the parameters $B_{i}, \quad i=1,2,3$ and the wave speed $c$ in such way that the boundary conditions (41) are satisfied.

To this end, we first observe that, on the basis of relations (42), (46) and (49), we must have

$$
c^{2}<v_{s}^{2} \text { and } X_{1,2}^{2}<\kappa^{2}
$$

and therefore, $\sigma_{s}$ and $\sigma_{p_{1,2}}$ are positive real numbers.

To satisfy the second condition in (53), it is necessary and sufficient to have

$$
\begin{aligned}
& c^{2}<\mathfrak{c}_{1}^{2} \text { and } \\
& 0<c^{4} \kappa^{2} \varrho^{2} \varkappa-c^{2} \varrho\left[\xi+\kappa^{2} \varkappa(\lambda+2 \mu)+\kappa^{2} \alpha\right]-\beta^{2}+\xi(\lambda+2 \mu)+\kappa^{2} \alpha(\lambda+2 \mu),
\end{aligned}
$$

where

$$
\mathfrak{c}_{1}^{2}=\frac{(\lambda+2 \mu) \xi-\beta^{2}+2 \kappa^{2} \alpha(\lambda+2 \mu)}{\varrho \kappa^{2}[(\lambda+2 \mu) \varkappa+\alpha]} .
$$

Since

$$
[(\lambda+2 \mu) \varkappa-\alpha]^{2} \varrho \varkappa \kappa^{4}+2\left[2 \beta^{2} \varkappa-(\lambda+2 \mu) \xi \varkappa+\xi \alpha\right] \kappa^{2}+\xi^{2}>0
$$

for all values of $\kappa$, from the inequality $(54)_{2}$ it follows that

$$
c^{2}<\mathfrak{c}_{2}^{2} \quad \text { or } \quad c^{2}>\mathfrak{c}_{3}^{2},
$$

where

$$
\begin{aligned}
\mathfrak{c}_{2}^{2}= & \frac{1}{2 \kappa^{2} \varrho \varkappa}\left\{\xi+\kappa^{2}(\lambda+2 \mu) \varkappa+\kappa^{2} \alpha-\right. \\
& \left.-\sqrt{\left(\xi+\kappa^{2}(\lambda+2 \mu) \varkappa+\kappa^{2} \alpha\right)^{2}-4 \kappa^{2} \varkappa\left[\xi(\lambda+2 \mu)-\beta^{2}+\kappa^{2} \alpha(\lambda+2 \mu)\right]}\right\}, \\
\mathfrak{c}_{3}^{2}= & \frac{1}{2 \kappa^{2} \varrho \varkappa}\left\{\xi+\kappa^{2}(\lambda+2 \mu) \varkappa+\kappa^{2} \alpha+\right. \\
& \left.+\sqrt{\left(\xi+\kappa^{2}(\lambda+2 \mu) \varkappa+\kappa^{2} \alpha\right)^{2}-4 \kappa^{2} \varkappa\left[\xi(\lambda+2 \mu)-\beta^{2}+\kappa^{2} \alpha(\lambda+2 \mu)\right]}\right\} .
\end{aligned}
$$


On the other hand, for all values of $k$, we have

$$
\mathfrak{c}_{1}^{2}>\mathfrak{c}_{2}^{2} \text { and } \mathfrak{c}_{1}^{2}<\mathfrak{c}_{3}^{2} .
$$

From (54) and (59), we deduce that the wave speed $c$ is admissible if

$$
c^{2}<\min \left\{v_{s}^{2}, \mathfrak{c}_{2}^{2}\right\}
$$

With the aid of relations (46) and (49), we find that the boundary conditions (41) are satisfied if and only if the unknown constants $B_{i}$ are solutions of the following algebraic system

$$
\begin{array}{r}
\left(2-\frac{c^{2}}{v_{s}^{2}}\right) B_{1}+2 \beta \sqrt{1-\frac{X_{1}^{2}}{\kappa^{2}}} B_{2}+2 \beta \sqrt{1-\frac{X_{2}^{2}}{\kappa^{2}}} B_{3}=0 \\
2 \sqrt{1-\frac{c^{2}}{v_{p}^{2}}} B_{1}+\beta\left(2-\frac{c^{2}}{v_{s}^{2}}\right) B_{2}+\beta\left(2-\frac{c^{2}}{v_{s}^{2}}\right) B_{3}=0 \\
\sqrt{1-\frac{X_{1}^{2}}{\kappa^{2}}}\left(\frac{X_{1}^{2}}{\kappa^{2}}-\frac{c^{2}}{v_{p}^{2}}\right) B_{2}+\sqrt{1-\frac{X_{2}^{2}}{\kappa^{2}}}\left(\frac{X_{2}^{2}}{\kappa^{2}}-\frac{c^{2}}{v_{p}^{2}}\right) B_{3}=0
\end{array}
$$

where

$$
v_{p}=\sqrt{\frac{\lambda+2 \mu}{\varrho}} .
$$

Since we have assumed that $\beta \neq 0$ it follows that the above system has a nontrivial solution if and only if the wave speed $c$ satisfies the following equation

$$
\begin{gathered}
R(c) \equiv\left[\left(2-\frac{c^{2}}{v_{s}^{2}}\right)^{2}-4 \sqrt{\left(1-\frac{c^{2}}{v_{s}^{2}}\right)\left(1-\frac{X_{1}^{2}}{\kappa^{2}}\right)}\right] \sqrt{1-\frac{X_{2}^{2}}{\kappa^{2}}}\left(-\frac{c^{2}}{v_{p}^{2}}+\frac{X_{2}^{2}}{\kappa^{2}}\right)- \\
-\left[\left(2-\frac{c^{2}}{v_{s}^{2}}\right)^{2}-4 \sqrt{\left(1-\frac{c^{2}}{v_{s}^{2}}\right)\left(1-\frac{X_{2}^{2}}{\kappa^{2}}\right)}\right] \sqrt{1-\frac{X_{1}^{2}}{\kappa^{2}}}\left(-\frac{c^{2}}{v_{p}^{2}}+\frac{X_{1}^{2}}{\kappa^{2}}\right)=0 .
\end{gathered}
$$

In view of relation (19), when $c=0$, we have

$$
X_{1}^{2}=\frac{\beta^{2}-\xi(\lambda+2 \mu)}{\alpha(\lambda+2 \mu)}<0 \text { and } X_{2}^{2}=0 .
$$

That proves that $c=0$ is solution of the equation (63).

From (16) and (37) we deduce that the effect of porosity vanishes when

$$
\frac{X^{2}}{\kappa^{2}}=\frac{c^{2}}{v_{p}^{2}} .
$$


Because $X_{1}^{2} \neq X_{2}^{2}$ we can say that only one value of $X$ can have the property (65). Thus, when we don't have the effect of porosity, the equation (63) becomes the well known secular equation for Rayleigh surface waves:

$$
\left(2-\frac{c^{2}}{v_{s}^{2}}\right)^{2}=4 \sqrt{\left(1-\frac{c^{2}}{v_{s}^{2}}\right)\left(1-\frac{c^{2}}{v_{p}^{2}}\right)} .
$$

We have to outline that, in classical elasticity, the solutions of the secular equation for Rayleigh surface waves are established numerically by Rayleigh [29]. Jeffreys [30] and Hayes \& Rivlin [31] also have studied the secular equation. For the statement of the problem please see the book [28] and the recent works [32]-[39].

In the following we assume that

$$
v_{s}^{2} \leq v_{p}^{2}-\frac{\beta^{2}}{\varrho \xi} \quad \text { and } \quad v_{s}^{2} \leq \frac{\alpha}{\varrho \varkappa}
$$

Let remark that, in this case,

$$
v_{s}^{2}<\mathfrak{c}_{2}^{2}
$$

and

$$
\begin{aligned}
R\left(v_{s}\right)= & \left(1-\frac{v_{s}^{2}}{v_{p}^{2}}\right)\left[\left(1-\frac{T_{2}\left(v_{s}^{2} \kappa^{2}\right)}{\kappa^{2}}\right)^{1 / 2}-\left(1-\frac{T_{1}\left(v_{s}^{2} \kappa^{2}\right)}{\kappa^{2}}\right)^{1 / 2}\right]- \\
& -\left[\left(1-\frac{T_{2}\left(v_{s}^{2} \kappa^{2}\right)}{\kappa^{2}}\right)^{3 / 2}-\left(1-\frac{T_{1}\left(v_{s}^{2} \kappa^{2}\right)}{\kappa^{2}}\right)^{3 / 2}\right] .
\end{aligned}
$$

From (19) and (29), we have

$$
T_{1}\left(v_{s}^{2} \kappa^{2}\right)+T_{2}\left(v_{s}^{2} \kappa^{2}\right)=-\frac{\xi(\lambda+2 \mu)-\beta^{2}-(\alpha+\varkappa(\lambda+2 \mu)) \varrho v_{s}^{2} \kappa^{2}}{\alpha(\lambda+2 \mu)}
$$

and hence the relation (69) becomes

$$
\begin{aligned}
& R\left(v_{s}\right)=\left[\left(1-\frac{T_{2}\left(v_{s}^{2} \kappa^{2}\right)}{\kappa^{2}}\right)^{1 / 2}-\left(1-\frac{T_{1}\left(v_{s}^{2} \kappa^{2}\right)}{\kappa^{2}}\right)^{1 / 2}\right]\left[-1-\frac{v_{s}^{2}}{v_{p}^{2}}-\right. \\
& \left.-\frac{\xi(\lambda+2 \mu)-\beta^{2}-(\alpha+\varkappa(\lambda+2 \mu)) \varrho v_{s}^{2} \kappa^{2}}{\alpha(\lambda+2 \mu)}-\left(1-\frac{T_{2}\left(v_{s}^{2} \kappa^{2}\right)}{\kappa^{2}}\right)^{1 / 2}\left(1-\frac{T_{1}\left(v_{s}^{2} \kappa^{2}\right)}{\kappa^{2}}\right)^{1 / 2}\right] .
\end{aligned}
$$

In view of assumptions (67), we have that the above $R\left(v_{s}\right)$ is a product of two negative numbers and thus $R\left(v_{s}\right)>0$. 
If we calculate $R\left(\varepsilon v_{s}\right)$, where $\varepsilon$ is a very small number, we find

$$
\begin{aligned}
R\left(\varepsilon v_{s}\right)=- & \frac{4 \beta^{2}}{(\lambda+2 \mu) \xi-\beta^{2}}\left(-1+\sqrt{1+\frac{(\lambda+2 \mu) \xi-\beta^{2}}{(\lambda+2 \mu) \alpha \kappa^{2}}}\right)+ \\
& \left.+\frac{2\left[-\beta^{2}+\varrho \xi\left(v_{p}^{2}-v_{s}^{2}\right)\right]}{\varrho \kappa^{2} v_{s}^{2} \alpha} \sqrt{1+\frac{(\lambda+2 \mu) \xi-\beta^{2}}{(\lambda+2 \mu) \alpha \kappa^{2}}}\right\} \varepsilon^{2},
\end{aligned}
$$

which is always negative. Hence equation (63) has at least one real root lying between 0 and $v_{s}$ if the conditions (67) are satisfied.

\section{References}

[1] M.Hayes, Inhomogeneous plane waves, Arch. Rational Mech. Anal. 85 (1984) 41-79.

[2] Ph. Boulanger, M. Hayes, Bivectors and Waves in Mechanics and Optics, Chapman \& Hall (1993).

[3] S.C. Cowin, J.W. Nunziato, Linear elastic materials with voids, J. Elasticity 13 (1983) 125147.

[4] M.A. Goodman, S.C. Cowin, A continuum theory for granular materials, Arch. Rational Mech. Anal. 44 (1972) 249-266.

[5] J.W. Nunziato, S.C. Cowin, A nonlinear theory of elastic materials with voids, Arch. Rational Mech. Anal. 72 (1979) 175-201.

[6] D. Ieşan, A theory of thermoelastic materials with voids, Acta Mechanica 60 (1986) 67-89.

[7] M. Ciarletta, D. Ieşan, Non-classical elastic solids, Longman Scientific \& Technical (1993).

[8] D. Ieşan, Thermoelastic models of continua, Kluwer Academic Publishers (2004).

[9] R. Quintanilla, On uniqueness and continuous dependence in the nonlinear theory of mixtures of elastic solids with voids, Math. Mech. Solids 6 (2001) 281-298.

[10] P.S. Casas, R. Quintanilla, Exponential decay in one-dimensional porous thermoelasticity, Mech. Res. Comm. 32 (2005) 652-658.

[11] A. Magaña, R. Quintanilla, On the time decay of solutions in one-dimensional theories of porous materials, Int. J. Solids Struct. 43 (2006) 3414-3427.

[12] A. Magaña, R. Quintanilla, On the spatial behaviour of solutions for porous elastic solids with quasi-static microvoids, Math. Comput. Modelling 44 (2006) 710-716.

[13] S. Chiriţă, I.D. Ghiba, Strong ellipticity and progressive waves in elastic materials with voids, Proc. R. Soc. London A 466 (2010), 439-458. 
[14] J.W. Nunziato, E.K. Walsh, On the influence of void compaction and material nonuniformity on the propagation of one-dimensional acceleration waves in granular materials, Arch. Rational Mech. Anal. 64 (1977) 299-316.

[15] J.W. Nunziato, E.K. Walsh, Addendum "On the influence of void compaction and material non-uniformity on the propagation of one-dimensional acceleration waves in granular materials", Arch. Rational Mech. Anal. 67 (1978) 395-398.

[16] J.W. Nunziato, J.E. Kennedy, E.K. Walsh, The behavior of one-dimensional acceleration waves in an inhomogeneous granular solid, Int. J. Eng. Sci. 16 (1978) 637-648.

[17] M. Ciarletta, B. Straughan, Poroacoustic acceleration waves, Proc. R. Soc. Lond. A 462 (2006) 3493-3499.

[18] M. Ciarletta, B. Straughan, Poroacoustic acceleration waves with second sound. J. Sound and Vibration 306 (2007) 725-731.

[19] M. Ciarletta, B. Straughan, V. Zampoli, Thermo-poroacoustic acceleration waves in elastic materials with voids without energy dissipation, Int. J. Eng. Sci. 45 (2007) 736-743.

[20] B. Straughan, Stability and wave motion in porous media, Springer (2008).

[21] M. Ostoja-Starzewski, J. Trebicki, On the growth and decay of acceleration waves in random media, Proc. R. Soc. Lond. A 455 (1999) 2577-2614.

[22] P. Puri, P.M. Jordan, On the propagation of plane waves in type-III thermoelastic media, Proc. R. Soc. Lond. A 460 (2004) 3203-3221.

[23] R. Quintanilla, B. Straughan, Discontinuity waves in type III thermoelasticity, Proc. R. Soc. Lond. A 460 (2004) 1169-1175.

[24] C.I. Christov, P.M. Jordan, Heat conduction paradox involving second sound propagation in moving media, Phys. Rev. Lett. 94 (2005) 154301-1-154301-4.

[25] P.M. Jordan, C.I. Christov, A simple finite difference scheme for modelling the finite-time blow-up of acoustic acceleration waves, J. Sound Vibration 281 (2005) 1207-1216.

[26] P.M. Jordan, P. Puri, Growth/decay of transverse acceleration waves in nonlinear elastic media, Phys. Lett. A 341 (2005) 427-434.

[27] I. Christov, P.M. Jordan, C.I. Christov, Nonlinear acoustic propagation in homentropic perfect gases: A numerical study, Phys. Lett. A 353 (2006) 273-280.

[28] J.D. Achenbach, Wave Propagation in Elastic Solids, North-Holland Publishing Company (1973).

[29] Lord Rayleigh, On Waves Propagated along the Plane Surface of an Elastic Solid, Proc. London Math. Soc. 17 (1885) 4-11.

[30] H. Jeffreys, The Earth, Cambridge University Press (1952).

[31] M. Hayes, S.R. Rivlin, A Note on the Secular Equation for Rayleigh Waves, ZAMP 13 (1962) $80-83$. 
[32] M. Rahman, J.R. Barber, Exact expression for the roots of the secular equation for Rayleigh waves, ASME J. Appl. Mech. 62 (1995) 250-252.

[33] D. Nkemzi, A new formula for the velocity of Rayleigh waves, Wave Motion 26 (1997) 199-205.

[34] P.G. Malischewsky, Comment to "A new formula for velocity of Rayleigh waves" by D. Nkemzi [Wave Motion 26 (1997) 199-205], Wave Motion 31 (2000) 93-96.

[35] P.C. Vinh, R.W. Ogden, On formulas for the Rayleigh wave speed, Wave Motion 39 (2004) 191-197.

[36] X.-F. Li, On approximate analytic expressions for the velocity of Rayleigh waves, Wave Motion 44 (2006) 120-127.

[37] P.C. Vinh, P.G. Malischewsky, An approach for obtaining approximate formulas for the Rayleigh wave velocity, Wave Motion 44 (2007) 549-562.

[38] P.C. Vinh, P.G. Malischewsky, Improved Approximations of the Rayleigh Wave Velocity, Journal Of Thermoplastic Composite Materials 21 (2008) 337-352.

[39] D. Nkemzi, A simple and explicit algebraic expression for the Rayleigh wave velocity, Mechanics Research Communications 35 (2008) 201-205. 